\title{
DETERMINACIÓN DE UN PATRÓN DE CALIDAD PARA LOS VINOS DE FRUTAS EN EL PERÚ
}

Carlos E. Tasayco Torres ', Víctor Echegaray Munenaka ${ }^{2}$, Luis Medina Marroquín ${ }^{3}$.

\section{RES U MEN}

Se encontró un patrón de calidad para los vinos de frutas en el Perú, realizando análisis físico-químicos, organolépticos y microbiológicos. Las características encontradas son concordantes con la norma técnica para vino de fruta del Instituto Colombiano de Normas Técnicas (ICONTEC 708, 1978), con excepción del grado alcohólico para vino de durazno $\left(6.4^{\circ} \mathrm{GL} 20^{\circ} \mathrm{C} / 20^{\circ} \mathrm{C}\right)$.

\begin{abstract}
A B S TR ACT
A pattern of quality wines in Peru was found, for which physicalchemical, organoleptic and microbiological analyses were carried out. The characteristics which were found are in accordance with the technical norm for fruit wine of the Instituto Colubiano de Normas Técnicas (ICONTEC 708, 1978 ) with the exception of the alcholic percentage of peaach wine (6.4' GL $20^{\circ} \mathrm{C} / 20^{\circ} \mathrm{C}$ ).
\end{abstract}

1. M.S.C. Ing. Industrias Alimentarias - Tecnología de Alimentos.

2. Mg. en Estadística.

3. Ing. en Industrias Alimentarias. 


\section{INTRODUCCIÓN}

En nuestro país, los vinos de fruta se comercializan sin tener un control físico-químico, microbiológico y organoléptico, ya que no se cuenta con una norma técnica que establezca las condiciones generales y requisitos que deben cumplir estos vinos de frutas, como sucede en otros países, caso de Colombia o Ecuador, que tienen normas técnicas para fijar la calidad de la bebida y garantizar la salud del consumidor. Se debe tener en cuenta que hace falta fijar los límites que han de imponerse a estos vinos, para así aprovechar sus numerosas virtudes y considerar por regla general que el consumidor debe tener la garantía de que está bebiendo un producto inocuo.

\section{OBJETIVO}

En el presente trabajo de investigación se pretende presentar una alternativa de solución, mediante el establecimiento de un patrón de calidad para dichos vinos de frutas en nuestro país.

\section{III.REVISIÓN BIBLIOGRÁFICA}

\section{VINO DE FRUTAS}

Según el Instituto Colombiano de Normas Técnicas (ICONTEC, 1978), vino de fruta es la bebida proveniente de mostos de frutas secas distintas de la uva, sometida a la fermentación alcohólica y que ha sufrido un proceso semejante a los exigidos para los vinos.

Según Vogt (1972), la elaboración de vinos de frutas no difiere de la vinificación de la uva, en lo que respecta al procedimiento de elaboración propiamente dicho y al tratamiento en las bodegas (sulfuración, clasificación, filtración y el embotellado de las mismas).

Para obtener el mosto de frutas, se realizaron acondicionamientos según la fruta. Brémond (1966) y Vogt (1972) coinciden en señalar que las principales correcciones que se realizan en el mosto, con la finalidad de mejorarlo son: corrección de azúcar, acidez y nutrientes, indicándose que todas las normas técnicas de vinos de frutas, permiten la adición de azúcar para obtener los grados alcohólicos requeridos; pero a la vez limitan la cantidad y no autorizan agregar azúcar al mosto ya fermentado.

\section{IV.NORMA TÉCNICA PARA VINOS DE FRUTA}

Según el ICONTEC (1978), los vinos de fruta deben cumplir las características siguientes : a) Características químicas: Los vinos de frutas podrán contener como máximo $18^{\circ} \mathrm{GL}$ de alcohol y mínimo $10^{\circ} \mathrm{GL}$; la acidez volátil, expresada como ácido acético $\left(\mathrm{g} / 100 \mathrm{~cm}^{3}\right)$, será como máximo 0.14 ; la acidez total expresada como ácido tartárico ( $\mathrm{g} /$ $100 \mathrm{~cm}^{3}$ ) debe oscilar entre 0.98 (máximo) y 0.5 (mínimo); asimismo, podrán contener ácido sórbico o sus sales en una cantidad máxima de 150 ppm y además, no se permitirá la presencia de otros preservativos, ni la adición de colorantes no autorizados.

b) Características organolépticas: Los vinos de frutas deben tener sabor, olor y color característicos, que dependen de la variedad de frutas, del proceso de fermentación y del añejamiento. Sin sabores ni olores extraños a la naturaleza propia de un vino de fruta sana.

c) Características microbiológicas: Deben estar libres de insectos, de microorganismos o de cualquier otra entidad capaz de causar la alteración del producto.

\section{MATERIALES Y MÉTODOS}

\section{MATERIALES}

Se utilizaron duraznos, plátanos, pera, melones, naranjas, piñas y ciruelas. Como medios de cultivo se emplearon caldo de extracto de levadura, solución salina peptonada, oxitetraciclina, glucosa, y los equipos: computadora, refractómetro, pulpeadora, alcoholímetro, equipo de destilación, estufa, espectrofotómetro, mufla, destilador semimicrokejdahl y espectrofotómetro de llama.

\section{MÉTODOS}

a) Analísis físico-químico: Humedad, materia seca, nitrógeno, fósforo y potasio (AOAC, 1960); densidad, sólidos solubles, acidez total, fija y volátil, suma de alcohol más acidez fija, relación alcohol/extracto seco reducido, cloruros, anhidrido sulfuroso total, libre y combinado, prueba al aire y quiebra cuprosa (NégretFrancot, 1980); azúcares reductores, reductores totales, sacarosa, grado alcohólico, alcoholes superiores, furfural sulfatos (ITINTEC, 1980); esteres y aldehídos (COPANT, 1971); extracto seco, cenizas totales, alcalinidad de las cenizas, bitartrato de $\mathrm{K}$ y acidez tartárica total (ministerio de Agricultura, 1973). 
b) Análisis organoléptico: Mediante una escala para vinos, según el ministerio de Agricultura y Alimentación (1973).

c) Análisis microbiológicos: Mohos y levaduras (Mossel y Quevedo, 1970), acetobacter (Becton Dickinson S.A., 1971).

d) Análisis estadístico: Aleatorio para determinar intervalos de aceptación, agrupados en valores semejantes, hallándose la media aritmética y desviación estándar.

\section{RESULTADOS Y DISCUSIÓN}

\section{CARACTERIZACIÓN DE LOS VINOS DE FRUTA}

En los cuadros 1A, 1B, 1C, se muestran los resultados de los vinos de durazno, piña, melón, pera, plátano, naranja, ciruela, donde se observa que todos los vinos de frutas estudiados cumplen con las normas técnicas para vinos de fruta, ICONTEC 708 Colombia, a excepción del grado alcohólico $\left(6.4^{\circ} \mathrm{GL} 20^{\circ} \mathrm{C} / 20^{\circ} \mathrm{C}\right)$ del vino de durazno. Se debe tener en cuenta que en el Perú no hay norma técnica para vinos de frutas, por lo que la comparación se hace con la norma técnica de un país vecino como es Colombia, cuya diversidad de frutales es muy parecida a nuestro medio.

Se debe indicar que los constituyentes químicos de los vinos, son útiles para diferenciar los tipos de vino, así como determinar su calidad, de acuerdo a las normas legales y medir su cantidad de componentes deseable e indeseable.

En los cuadros 2 y 3 se muestran los resultados de las evaluaciones organolépticas y microbiológicas, respectivamente, de los vinos de frutas estudiados. De acuerdo a estos resultados, los vinos de frutas fueron aceptados por los panelistas, organolépticamente, con la calificación de calidad: sabor: bueno (31.9), aroma y bouquet: bueno (20.4), color y transparencia: muy bueno (16.04). El control microbiológico nos indica que hubo ausencia de mohos, levaduras y acetobacter en los vinos de piña, ciruela, pera, plátano y melón; no sucediendo lo mismo con los vinos de durazno y naranja, debido a que el vino de naranja se elaboró a partir de naranja sobremadura y que durante las pasteurizaciones y procesos de elaboración, no se tuvo mucho cuidado; aunque los valores obtenidos son muy bajos.

\section{CUADRO № 1A: Características fisico-químicas de los vinos de durazno y piña.}

\begin{tabular}{|c|c|c|}
\hline \multirow[b]{2}{*}{ CARACTERÍSTICAS } & \multicolumn{2}{|c|}{ VINOS } \\
\hline & DURAZNO & PIÑA \\
\hline Grado alcohólico ${ }^{\circ} \mathrm{GL} 20^{\circ} \mathrm{C} / 20^{\circ} \mathrm{C}$ & 6.480 & 13.000 \\
\hline Densidad & 1.015 & 1.026 \\
\hline $\mathrm{pH}$ & 3.100 & 3.400 \\
\hline Sólidos solubles ( ${ }^{\circ}$ Brix) & 5.000 & 12.700 \\
\hline Acidez total g. ácido sulfúrico/l & 3.750 & 4.507 \\
\hline Acidez fija g ácido sulfúrico/l & 3.401 & 4.196 \\
\hline Acidez volátil g ácido acético/l & 0.429 & 0.430 \\
\hline Alcohol + acidez fija & 9.881 & 17.196 \\
\hline Extracto seco $\mathrm{g} / \mathrm{l}$ & 105.730 & 102.130 \\
\hline Azúcares reductores g glucosa $A$ & 35.100 & 74.100 \\
\hline Reductores totales g glucosa/ & 99.600 & 149.102 \\
\hline Sacarosa $g / 1$ & 65.300 & 67.547 \\
\hline Relación alcohol/extracto seco reduф & 0.945 & 4.934 \\
\hline Ésteres g. acetato de etilo/l & 0.085 & 0.281 \\
\hline Alcoholes superiores $\mathrm{mg} / 100 \mathrm{ml}$ & 10.200 & 0.250 \\
\hline Furfural $\mathrm{mg} / 100 \mathrm{ml}$ & 0.049 & 0.346 \\
\hline Fósforo g/l & 0.050 & $\ldots$ \\
\hline Potasio $\mathrm{g} / \mathrm{l}$ & 0.170 & $\cdots+$. \\
\hline Nitrógeno g/l & 0.150 & $\cdots$ \\
\hline Bitartrato de potasio $\mathrm{g} / \mathrm{l}$ & $\cdots$ & 0.425 \\
\hline Acidez tartárica total g acid. tart// & $\cdots$ & 0.341 \\
\hline Cenizas $\mathrm{g} / \mathrm{l}$ & 2.545 & 2.940 \\
\hline Alcalinidad de las cenizas $\mathrm{g} \mathrm{K} 2 \mathrm{CO}_{3}$ & $\ldots+.$. & 1.518 \\
\hline Anhidrido sulfuroso total $\mathrm{g} \mathrm{SO}_{2} \mathrm{~A}$ & 0.158 & 0.140 \\
\hline Anhidrido sulfuroso libre $\mathrm{g} \mathrm{SO}_{2} /$ & 0.068 & 0.053 \\
\hline Fierro $\mathrm{mg} / \mathrm{l}$ & 2.200 & -.... \\
\hline Calcio mg/l & 0.032 & ..... \\
\hline
\end{tabular}

\section{INTERVALOS DE ACEPTACIÓN DE LOS VINOS DE FRUTAS}

En el cuadro 4 se reportan los intervalos de aceptación para los distintos componentes de los vinos de frutas en el Perú. De acuerdo a estos resultados, los grados alcohólicos y densidad están en un rango de 9.35 a $15.04^{\circ} \mathrm{GL} 20^{\circ} \mathrm{C} / 20^{\circ} \mathrm{C}$ y 0.983 a 1.020 , respectivamente. Se puede notar que el rango de grado alcohólico llega hasta $15.04^{\circ} \mathrm{GL}$, debido a que se permite agregar azúcar al mosto de fruta; pero que tiene un limite que, según ICONTEC (1978), debe ser hasta 160 $\mathrm{g} / \mathrm{dm}^{3}$, y los grados alcohólicos finales no deben exceder de $18^{\circ} \mathrm{GL}$ y no ser menor de $10^{\circ} \mathrm{GL}$, lo que ha sucedido en los vinos de frutas estudiados, a excepción del vino de durazno que tiene $1.4^{\circ} \mathrm{GL}$. 
CUADRO № 1B: Características físico-quimicas de los vinos de pera, melón y plátano.

\begin{tabular}{|c|c|c|c|}
\hline \multirow[b]{2}{*}{ CARACTERISTICAS } & \multicolumn{3}{|c|}{ VINOS } \\
\hline & PERA & MELÓN & PLÁTAN \\
\hline Grado alcohólico ${ }^{\circ} \mathrm{GL} 20^{\circ} \mathrm{C} / 20^{\circ} \mathrm{C}$ & 12.000 & 12.410 & 15.000 \\
\hline Densidad & 0.998 & 0.983 & -........ \\
\hline $\mathrm{pH}$ & 3.600 & 3.830 & ……- \\
\hline Sólidos solubles ( ${ }^{\circ}$ Brix) & 9.800 & 9.900 & -n...... \\
\hline Acidez total g. ácido sulfúrico/l & 2.978 & 1.997 & .......... \\
\hline Acidez fija g. ácido sulfúrico/l & 2.642 & 1.604 & .........- \\
\hline Acidez volátil g. ácido acético/l & 0.411 & 0.481 & 0.064 \\
\hline Alcohol + acidez fija & 15.440 & 14.010 & n........ \\
\hline Extracto seco g/l & 56.390 & 59.370 & $\cdots+\cdots$ \\
\hline Azúcares reductores g. glucosa / & 14.170 & 13.730 & $\cdots+\cdots . .$. \\
\hline Reductores totales g. glucosall & 19.980 & 20.030 & -........ \\
\hline Sacarosa $\mathrm{g} / \mathrm{l}$ & 5.510 & 6.520 & -....... \\
\hline Relación alcohol/extracto seco redy & c. 3.200 & $3.190^{\circ}$ & -....... \\
\hline Cloruros g. $\mathrm{NaCl} / \mathrm{I}$ & 0.610 & 0.380 & (n........ \\
\hline Sulfatos g. $\mathrm{K}_{2} \mathrm{SO}_{4} / \mathrm{I}$ & 0.480 & 0.500 & ….... \\
\hline Aldehídos g.acetaldehido/l & 0.300 & 0.410 & 0.647 \\
\hline Alcoholes superiores $\mathrm{mg} / 100 \mathrm{ml}$ & 6.520 & 5.430 & -........ \\
\hline Furfural $\mathrm{mg} / 100 \mathrm{ml}$ & trazas & -........ & .......... \\
\hline Fósforo g/l & 0.600 & 0.560 & ......... \\
\hline Nitrógeno g/l & 0.090 & 0.080 & …-... \\
\hline Acidez tartárica total g.ácid. tart/l & 0.110 & 0.060 & -..-.... \\
\hline Cenizas g/l & 4.430 & 5.160 & -..-..... \\
\hline Alcalinidad de las cenizas g. $\mathrm{S}_{2} \mathrm{CO}_{3}$ & 11.118 & 0.820 & …..... \\
\hline Anhidrido sulfuroso total g. $\mathrm{SO}_{2} / 1$ & 0.083 & 0.160 & …..... \\
\hline Anhidrido sulfuroso libre $\mathrm{gSO}_{2} /$ & 0.022 & 0.030 & 0.020 \\
\hline Anhidrido sulfuroso combinado g. $\mathrm{SO}_{2}$ A & 0.060 & 0.120 & …..... \\
\hline Quiebra cuprosa & Buena & Buena & -.n-.... \\
\hline Prueba al aire & Buena & Buena & -....... \\
\hline Humedad $(\%)$ & 94.090 & 94.870 & -.......- \\
\hline Materia seca $(\%)$ & 5.900 & 5.123 & -...-...- \\
\hline Ésteres g. acetaldehido/l & 0.350 & -....... & ......... \\
\hline Potasio g/l & 3.980 & ........ & -....... \\
\hline Bitartrato de potasio g/ & 0.990 & $\cdots$ & -....... \\
\hline
\end{tabular}

El pH oscila entre 3,24 y 3,83 , lo que nos da una pauta de protección de los vinos de frutas, ya que si fueran mayores, estarían a expensas de microorganismos no deseados para una buena conservación. Los sólidos solubles están entre 6,90 y $11,8^{\circ}$ Brix, lo que nos indica que los vinos de frutas tienen un cierto dulzor y contienen azúcares.

Los resultados de la acidez total y acidez fija, oscilan
CUADRO № 1C: Características físico-quimicas de los vinos de naranja y ciruela.

\begin{tabular}{|c|c|c|}
\hline \multirow[b]{2}{*}{ CARACTERÍSTICAS } & \multicolumn{2}{|c|}{ VINOS } \\
\hline & NARANJA & CIRUELA \\
\hline Grado alcohólico ${ }^{\circ} \mathrm{GL} 20^{\circ} \mathrm{C} / 20^{\circ} \mathrm{C}$ & 14.600 & 11.130 \\
\hline Densidad & 0.980 & 1.012 \\
\hline $\mathrm{pH}$ & 3.900 & 3.430 \\
\hline Sólidos solubles $\left({ }^{\circ}\right.$ Brix $)$ & 9.600 & 9.130 \\
\hline Acidez total g. ácido sulfúrico/l & 8.450 & 4.350 \\
\hline Acidez fija g. ácido sulfúrico/I & 8.180 & 4.090 \\
\hline Acidez volátil g. ácido acético/l & 0.320 & 0.310 \\
\hline Alcohol + acidez fija & 22.700 & 15.223 \\
\hline Extracto seco g/l & 29.400 & 113.830 \\
\hline Azúcares reductores g. glucosa / & 66.870 & 39.147 \\
\hline Reductores totales g. glucosa/l & 18.450 & 134.300 \\
\hline Sacarosa g/l & 1.500 & 90.400 \\
\hline Relación alcohol/extracto seco reduc. & 5.080 & 1.710 \\
\hline Cloruros g. $\mathrm{NaCl} / \mathrm{I}$ & 0.190 & 0.270 \\
\hline Sulfatos g. $\mathrm{K}_{2} \mathrm{SO}_{4} / \mathrm{I}$ & 0.128 & 0.336 \\
\hline Ésteres g. acetato de etilo/l & 0.130 & 0.370 \\
\hline Aldehidos g. acetaldehido/l & 0.220 & 0.310 \\
\hline Alcoholes superiores $\mathrm{mg} / 100 \mathrm{ml}$ & 0.300 & 9.770 \\
\hline Furfural $\mathrm{mg} / 100 \mathrm{ml}$ & 0.002 & 0.005 \\
\hline Fósforo g/l & 0.070 & 0.250 \\
\hline Potasio g/l & 0.907 & 1.400 \\
\hline Nitrógeno g/l & 0.086 & 0.337 \\
\hline Bitartrato de potasio g/l & 0.907 & 0.381 \\
\hline Acidez tartáriça total g.ácid. tart/l & 0.143 & 0.294 \\
\hline Cenizas g/l & 7.400 & 3.770 \\
\hline Alcalinidad de las cenizas g. $\mathrm{K}_{2} \mathrm{CO}_{3} / \mathrm{I}$ & 0.590 & 1.280 \\
\hline Anhidrido sulfuroso total $\mathrm{g} \cdot \mathrm{SO}_{2} \mathrm{n}$ & 0.017 & 0.051 \\
\hline Anhidrido sulfuroso libre $\mathrm{g} \cdot \mathrm{SO}_{2} /$ & 0.009 & 0.003 \\
\hline Anhidrido sulfuroso combinado g. $\mathrm{SO}_{2} 1$ & 0.007 & 0.047 \\
\hline Quiebra cuprosa & Buena & Buena \\
\hline Prueba al aire & Buena & Buena \\
\hline Fierro $\mathrm{mg} / \mathrm{l}$ & 1.840 & ........ \\
\hline Cobre $\mathrm{mg} / \mathrm{l}$ & 0.360 & $\cdots$ \\
\hline Zinc $\mathrm{mg} /$ & 1.120 & -...-...- \\
\hline Calcio g/l & 0.122 & ….... \\
\hline Magnesio mg/ & 5.200 & -....... \\
\hline Sodio $\mathrm{mg} / \mathrm{l}$ & 616.000 & 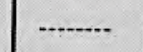 \\
\hline Vitamina C mg/l & 2.150 & $+\cdots+. .$. \\
\hline Manganeso $\mathrm{mg} / \mathrm{l}$ & 0.300 & 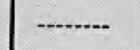 \\
\hline
\end{tabular}

entre 2,107 a 6,552 y 1,760 a 6,279 g ácido sulfúrico/ L respectivamente, que están dentro del rango especificado para vinos de frutas. Los datos de acidez 


\section{CUADRO № 2: Evaluación organoléptica de los vino de frutas.}

\begin{tabular}{|l|l|c|c|}
\hline \multicolumn{4}{|c|}{ FACTORES DE CALIDAD EVALUADOS } \\
\hline VINOS & SABOR & $\begin{array}{c}\text { AROMA Y } \\
\text { BOUQUET }\end{array}$ & $\begin{array}{l}\text { COLOR Y } \\
\text { TRANSPARENCIA }\end{array}$ \\
\hline Durazno & 37.0 & 19.8 & 18.8 \\
Piña & 30.0 & 16.8 & 10.0 \\
Naranja & 31.0 & 24.6 & 17.0 \\
Ciruela & 31.5 & 20.4 & 17.6 \\
Pera & 30.0 & 20.4 & 16.8 \\
\hline PROMEDIO & 31.9 & 20.4 & 16.04 \\
\hline
\end{tabular}

CUADRO № 3: Resultado microbiológico de los vinos de frutas.

\begin{tabular}{|l|r|r|c|}
\hline VINOS & $\begin{array}{c}\text { MOHOS } \\
\text { (ufc/ml) }\end{array}$ & $\begin{array}{r}\text { LEVADURA } \\
\text { (ufc/ml) }\end{array}$ & $\begin{array}{r}\text { ACETOBÁCTER } \\
\text { (ufc/ml) }\end{array}$ \\
\hline Durazno & 1 & 1 & 1 \\
Piña & 0 & 0 & 0 \\
Naranja & 100 & 100 & 100 \\
Ciruela & 0 & 0 & 0 \\
Pera & 0 & 0 & 0 \\
Melón & 0 & 0 & 0 \\
Plátano & 0 & 0 & 0 \\
\hline
\end{tabular}

fija nos sirven para diferenciar los diverșos tipos de vino, los rangos encontrados están dentro de lo recomendado por Amerine y Joslyn (1970), quienes manifiestan que los vinos de fruta no deben tener una alta acidez fija, porque pueden adquirir un gusto ácido, amargo, indeseable. En lo que respecta a la acidez volátil $(0,21$ a 0,489 g ácido acético/ I), nos indica que los vinos de frutas son sanos y están lejos del máximo permitido para vinos de frutas, recomendado por ICONTEC (1978).

En cuanto al extracto seco $(43,70$ a $111,89 \mathrm{~g} . / \mathrm{l})$, los rangos varían grandemente, debido a que los vinos de pera, durazno, piña y ciruela tienen mayor extracto seco no así el vino de naranja. Según Amerine y Joslyn (1970), los vinos que tienen extracto seco bajo, son muy ligeros al paladar, comparados con los vinos que tienen extracto seco alto.

En lo referente a los ésteres, de acuerdo a los
CUADRO № 4: Intervalos de aceptación para los vinos de frutas en el Perú.

\begin{tabular}{|c|c|}
\hline CARACTERISTICAS & $\begin{array}{l}\text { INTERVALO DE } \\
\text { ACEPTACIÓN }\end{array}$ \\
\hline Grado alcohólico ${ }^{\circ} \mathrm{GL} 20^{\circ} \mathrm{C} / 20^{\circ} \mathrm{C}$ & $<9.350,15.040>$ \\
\hline Densidad & $<0.983,1.020\rangle$ \\
\hline $\mathrm{pH}$ & $<3.240,3.830>$ \\
\hline Sólidos solubles ( ${ }^{\circ}$ Brix $)$ & $<6.900,11.800>$ \\
\hline Acidez total g. ácido sulfúrico/L & $<2.107,6.552>$ \\
\hline Acidez fija g. ácido sulfúrico/L & $<1.760,6.279>$ \\
\hline $\begin{array}{l}\text { Acidez volátil g. ácido acético/L } \\
\text { Nitrógeno } \mathrm{g} / \mathrm{L}\end{array}$ & $\begin{array}{ll}<0.210, & 0.489 \\
<0.041, & 0.257>\end{array}$ \\
\hline Bitartrato de potasio $\mathrm{g} / \mathrm{L}$ & $\langle 0.357, \quad 0.992\rangle$ \\
\hline Acidez tartárica total g.ácid. tart/L & $\langle 0.070, \quad 0.310\rangle$ \\
\hline Cenizas $g / L$ & $<2.609,6.139>$ \\
\hline Alcalinidad de las cenizas g. $\mathrm{K} 2 \mathrm{CO}_{3} / \mathrm{L}$ & $<0.704,1.431>$ \\
\hline Anhidrido sulfuroso total g. $\mathrm{SO}_{2} / \mathrm{L}$ & $<0.040, \quad 0.164>$ \\
\hline Anhidrido sulfuroso libre $\mathrm{g} . \mathrm{SO}_{2} \mathrm{~L}$ & $<0.007, \quad 0.054>$ \\
\hline Anhidrido sulfuroso combinado g. $\mathrm{SO}_{2}$ & $L<0.027, \quad 0.110>$ \\
\hline Humedad $(\%)$ & $<93.81,95.05>$ \\
\hline Materia seca $(\%)$ & $<4.957, \quad 6.074>$ \\
\hline Fierro mg/L & $\langle 1.765,2.274>$ \\
\hline Calcio $\mathrm{g} / \mathrm{L}$ & $\langle 0.013, \quad 0.140\rangle$ \\
\hline
\end{tabular}

intervalos de aceptación $(0,114$ a 0,374 g. acetato de etilo/l), también han influenciado sobre la aceptación por parte de los panelistas, ya que según Amerine et al (1980), los ésteres, como el etil acetato, son considerados como un factor importante en el aroma y bouquet. Los rangos encontrados de aceptabilidad de ésteres están dentro de lo recomendado por Amerine (1970).

Los alcoholes superiores están en bajas cantidades, (rango de 0.879 a $10.611 \mathrm{mg} / 100 \mathrm{ml}$ ). Estas cantidades son inferiores a los presentes en los vinos de uva reportados por Reventos (1960). Por su parte, Ribereau - Gayón (1960), afirman que los alcoholes superiores constituyen la parte principal de las sustancias del bouquet.

Las cantidades de furfural ( 0.0013 a $0.0056 \mathrm{mg} /$ $100 \mathrm{ml}$ ) son mucho menores que los alcoholes superiores, lo que nos demuestra que los rangos obtenidos de aceptabilidad están garantizados, pues al furfural casi siempre se le asocia con los alcoholes superiores, porque un exceso de cualquiera de ellos, produce toxicidad en el organismo (Amerine, 1970).

El anhidrido sulfuroso total, encontrado en los vinos de fruta, está en un rango de 0,040 a 0,164 g. SO$/ /$. Estos intervalos de aceptabilidad están dentro del 
máximo permitido para vinos de frutas, que es de 0,25 g. $\mathrm{SO}_{2} /$, según ICONTEC (1978). El contenido de nitrógeno de los vinos de frutas $(0,041$ a $0,257 \mathrm{~g} / \mathrm{l})$, nos indica que todos ellos están dentro de una calidad aceptable, pudiéndoseles agrupar dentro de un rango. El contenido de fierro oscila entre 1.765 a $2.274 \mathrm{mg} / \mathrm{l}$, lo cual está dentro los límites recomendados por Amerine et al (1980). El fierro es de interés en la formación de opacidad, reacción de oxido-reducción y efectos de la levadura.

Los rangos de aceptabilidad de sulfatos y cloruros $\left(0,296\right.$ a $0,508 \mathrm{~g} \mathrm{~K}_{2} \mathrm{SO}_{4} / \mathrm{l}$ y 0,187 a $0,546 \mathrm{~g}$. NaCl/l, respectivamente) nos demuestran que están dentro de lo especificado por la norma técnica del ITINTEC-Perú para vinos, además, permite agrupar a todos los vinos de frutas, debido a que probablemente provienen de similares tipos de suelo, los cuales tienen fosfatos de amonio o sulfato de amonio.

\section{CONCLUSIONES}

1. Los vinos de fruta por sus características físicoquímicas, microbiológicas y organolépticas, pueden

\section{BIBLIOGRAFÍA}

Amerine M.A, 1970. Wine, Edit. Kirk Othmer, $2^{\text {da }}$ edic., New York.

Amerine, Berg Kinkee, Ough, Singleton, Webb, 1980. The Technology of wine making. Edit. Av Publishing.

Amerine y Joslyn, 1979. Table wine: The Technology of their production. California USA.

AOAC, 1960. Official Methods of Analysis of Asociation Agricultural Chemists. Edit. Boar, USA.

Brémond E., 1966. Técnicas Modernas de Vinificación y Conservación de Vinos. Edit. José Monteso. Barcelona - España.

Becton Dickinson S.A., 1971. Manual de Procedimientos de Laboratorios y Productos. B.B.L. Química Suiza, México.

COPANT, 1971. Normas Técnicas para Análisis de Bebidas Alcohólicas, № 1030, Argentina.

ICONTEC, 1978. Normas Técnicas para Vinos de Frutas, № 708 , Colombia. determinar intervalos de aceptación y por ende un patrón de calidad para los distintos componentes de los mismos.

2. Los vinos de frutas son aceptados organolépticamente como buenos en aroma, sabor y bouquet; muy buenos en color y transparencia.

3. Los vinos de frutas en el Perú cumplen con la norma técnica para vinos de frutas, ICONTEC 708 Colombia, a excepción de los grados alcohólicos del vino de durazno.

\section{RECOMENDACIONES}

1. EI INDECOPI debe fijar una norma técnica que establezca los requisitos de los vinos de frutas en el Perú, y para ello debe utilizar los intervalos de aceptación encontrados en los diferentes componentes de los vinos de frutas, logrados en el presente trabajo de investigación.

ITINTEC, 1980. Norma Técnica para Análisis de Vinos y Bebidas Alcohólicas. Lima-Perú.

Ministerio de Agricultura, 1973. Laboratorio Central de Control de Calidad Sección de Alcoholes, Vinos y Licores. Métodos de Análisis, Lima-Perú.

Mossel y Quevedo, 1967. Control Microbiológico de los Alimentos. Monografía de CLEIBA, Lima-Perú.

Negcet-Francot, 1980. Vinificación y Conservación de los Vinos. Edit. Monteso, España.

Reventos, 1960. Guía Práctica del Comerciante en Vinos. Edit. Sintes, España.

Ribereau-Gayón, 1960. Traite do énologia. Librairia Polytecnique Ch.,tomo I, Francia.

Vogt E., 1972. Fabricación de Vinos. Edit. Acribia, ZaragozaEspaña. 